\title{
Orientation Mapping by Precession Transmission Electron Microscopy
}

\author{
Danqi Wang ${ }_{2}^{1}$ Amir Avishai ${ }^{1}$, Arthur Heuer ${ }^{1,2}$
}

1. Swagelok Center for Surface Analysis of Materials, Case Western Reserve University, Cleveland, OH, USA.

2. Department of Materials Science and Engineering, Case Western Reserve University, Cleveland, OH, USA.

Precession transmission electron microscopy (PTEM) is currently a very "hot” topic [1]. One of its major applications is orientation mapping. While conventional electron backscattered diffraction (EBSD) and transmission Kikuchi diffraction (TKD) [2] rely on Kikuchi pattern analysis, PTEM (ASTAR system by Nanomegas) acquires micro-diffraction patterns for orientation information. Therefore, even though both methods work in most samples, it is possible that for certain samples PTEM may have an advantage over the EBSD/TKD technique.

Low-temperature nitridation of stainless steels introduces several GPa residual stresses due to a "colossal” interstitial nitrogen supersaturation [3], which introduces a significant lattice deformation. While Kikuchi diffraction is sensitive to the lattice distortion, the ASTAR electron micro-diffraction approach focuses on image matching, and is not sensitive to such distortions.

TKD was carried out on low-temperature nitrided ferrite in 17-7 PH stainless steel. As shown in Fig. 1, two ferrite grains were identified in the cross sectional TEM foil. As the sample free surface is to the left of the foil, the two ferrite grains are at different depths in the nitrided layer, and thus subject to different amount of deformation. For the ferrite grain deeper in the layer (on the right), which is subject to a lower deformation, TKD could identify almost the entire ferrite grain. However, the ferrite grain closer to the free surface (on the left) cannot be identified at all, due to a much higher lattice deformation. In the highly deformed ferrite grain, the Kikuchi pattern from the grain was completely smeared out and thus could not be indexed by the software. This suggests the limitation of TKD on the highly deformed microstructurse. Such deformation is more related to the microscopic lattice deformation, rather than the macroscopic deformation, such as White Etched Areas observed in low-carbon steels after dynamic loading conditions [5].

The same TEM foil was characterized by PTEM. Fig. 2 shows that both of the ferrite grains were successfully indexed. There are still regions in the ferrite cannot be indexed due to the distortion in diffraction patterns from the deformed ferrite grain. This is illustrated in the reliability map (Fig. 2b); the ferrite grain with higher deformation has a lower reliability compared to that of ferrite deeper in the material. Therefore, in the case of heavy deformed microstructures, PTEM has an advantage over TKD.

\section{References:}

[1] Brons JG, et al., JOM, 66 (2014), p. 165.

[2] Wu D, et. al., Acta Mater, 79 (2014), p. 339.

[3] Wang D, et al., Acta Mater, 86 (2015), p. 193.

[4] Keller RR, et al., J Micro 245 (2012), p. 245.

[5] Avishai et al., submitted to M\&M 2015. 
a.

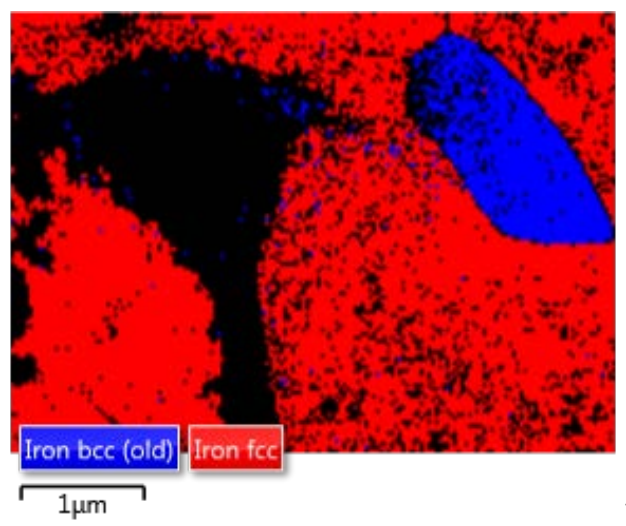

b.

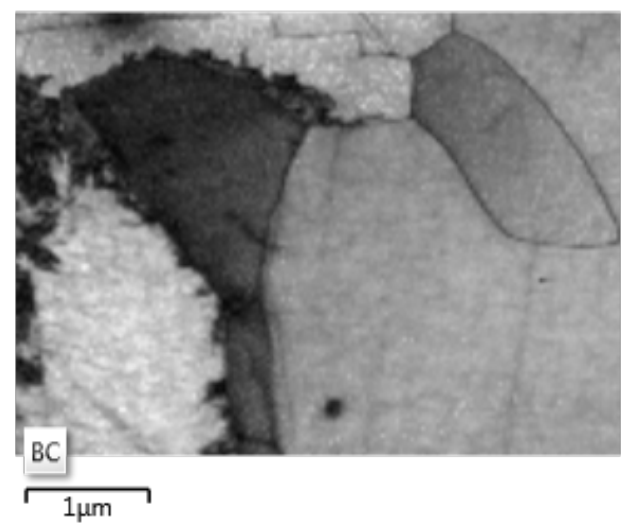

Figure 1. Data acquired by TKD from cross-sectional nitrided 17-7 PH stainless steel with free surface on the left. (a) Phase map and (b) Band contrast.

a.

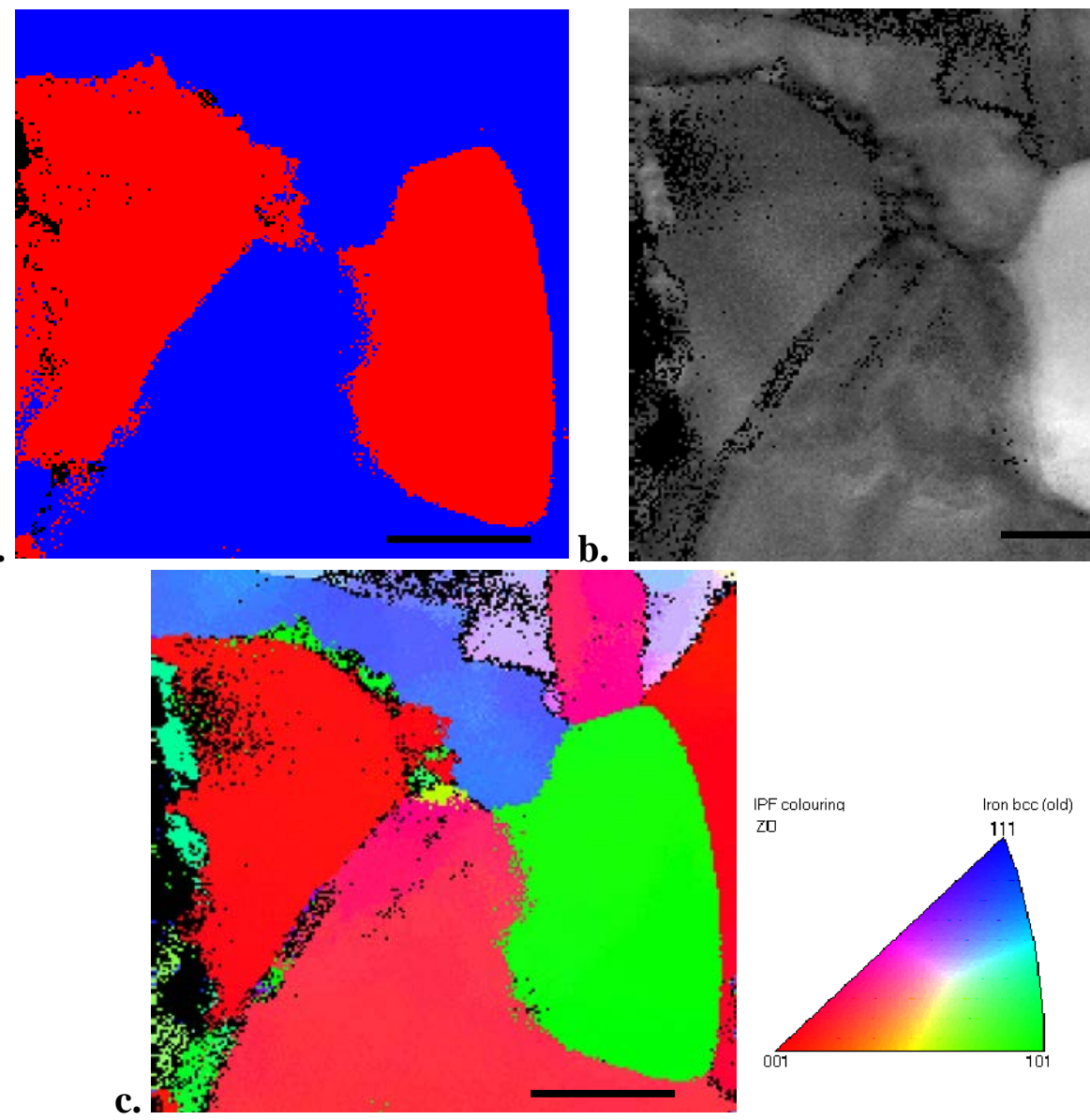

Figure 2. Data acquired by precessed electron beam in TEM of the same TEM foil in (a). (a) Phase map, (b) reliability map, and (c) orientation map. Scale bars in all images are $1 \mu \mathrm{m}$. 